\title{
Observer-based Guaranteed Cost Control for a Class of Singular Time-delay Systems with Uncertainties
}

\author{
Zhang $\mathrm{Na}$, Ma Yuechao, Liang Jianyun \\ College of Sciences \\ Yanshan University \\ Qinhuangdao Hebei, China
}

\begin{abstract}
The problem of guaranteed cost observer-based controller design for a class of singular systems time-delay with uncertainties is investigated. The design method of guaranteed cost observer-based controller is given. Based on linear matrix inequality (LMI) approach, sufficient conditions for the existence of guaranteed cost observer-based state feedback control law and corresponding guaranteed cost performance index are obtained by constructing generalized Lyapunov function. It makes that the closed-loop system is robust stable.
\end{abstract}

Keywords-Observer-based controller; guaranteed cost control; time-delay systems; linear matrix inequality

\section{INTRODUCTION}

The control of singular systems has been extensively studied in the past years[1-2]. In [3-4], guaranteed cost control for uncertain systems are discussed. In [5-6], observer-based guaranteed cost control for singular time-delay systems with uncertainties are discussed, but the state matrices and the control input matrices are consistent with the original system.

In this paper, we consider observer-based guaranteed cost control for a class of singular time-delay systems with uncertainties. The state matrices and the control input matrices are not consistent with the original system and are unknown in this paper. Based on linear matrix inequality (LMI) approach, sufficient conditions for the existence of guaranteed cost observer-based state feedback control law and corresponding guaranteed cost performance index are obtained by constructing generalized Lyapunov function. It makes that the closed-loop system is robust stable.

\section{PROBLEMS FORMULATION}

Consider the following uncertain singular time-delay systems

$E \dot{x}(t)=(A+\Delta A) x(t)+\left(A_{1}+\Delta A\right) x\left(t-d_{1}(t)\right)+(B+\Delta B) u(t)+\left(B_{1}+\Delta B\right) u\left(t-d_{2}(t)\right)$

$y(t)=(C+\Delta C) x(t)+\left(C_{1}+\Delta C_{1}\right) x\left(t-d_{1}(t)\right)+(D+\Delta D) u(t)+\left(D_{1}+\Delta D\right) u\left(t-d_{2}(t)\right)$ $x(t)=\varphi(t), t \in[-d, 0]$

(1)

Where $x(t) \in R^{n}$ is the state vector, $u(t) \in R^{n}$ is the control input vector, $E, A, A_{1}, B, B_{1}, C, C_{1}, D, D_{1}, C_{2}$ and $D_{2}$ are known real constant matrices with appropriate dimensions, where $E \in R^{n \times n}$ may be singular and we assume that
$\operatorname{rankE}=r \leq n ; \Delta A, \Delta A_{1}, \Delta B, \Delta B_{1}, \Delta C, \Delta C_{1}, \Delta D$ and $\Delta D_{1}$ are uncertain matrices representing time-varying parameter uncertainties in the system model, $d_{1}(t)$ and $d_{2}(t)$ are unknown constant matrices representing the number of delay units in the state and input respectively, which satisfy $0 \leq d_{1}(t)<d_{1}<\infty, 0 \leq d_{2}(t)<d_{2}<\infty$,

$d=\max \left[d_{1}, d_{2}\right], \quad d_{1}(t) \leq \alpha<1, \quad d_{2}(t) \leq \beta<1, \quad d_{1}(0)=\lambda ，$ $d_{2}(0)=\eta$, The parameter uncertainties considered in this paper are assumed to be norm-bounded and of the form

$$
\begin{aligned}
& \Delta A=E_{1} F_{1}(t) H_{1}, \Delta B=E_{2} F_{2}(t) H_{2}, \Delta A_{1}=E_{3} F_{3}(t) H_{3}, \Delta B_{1}=E_{4} F_{4}(t) H_{4}, \\
& \Delta C=E_{5} F_{5}(t) H_{5}, \Delta D=E_{6} F_{6}(t) H_{6}, \Delta C_{1}=E_{7} F_{7}(t) H_{7}, \Delta D_{1}=E_{8} F_{8}(t) H_{8},
\end{aligned}
$$

Where $E_{i}, H_{i}(i=1,2, \cdots, 10)$ are unknown real constant matrices with appropriate dimensions ,and $F_{i}(t) \quad(i=1,2, \cdots, 8)$ are unknown real matrices with Lebesgue-measurable elements and satisfy

$$
F_{i}^{T}(t) F_{i}(t) \leq I_{g_{i}}, i=1,2, \cdots, 8,
$$

We define the cost function:

$$
J=\int_{0}^{\infty}\left[x^{T}(t) Q x(t)+u^{T}(t) R u(t)\right] d t
$$

where $Q>0$ and $R>0$ are given matrices.

The objective is to design an observer-based controller of the form:

$$
\begin{aligned}
E \dot{\xi}(t) & =A_{C} \xi(t)+A_{D} \xi\left(t-d_{1}(t)\right)+B_{C} u(t)+B_{D} u\left(t-d_{2}(t)\right)+L[y(t)-\hat{y}(t)] \\
\hat{y}(t) & =C \xi(t)+C_{1} \xi\left(t-d_{1}(t)\right)+D u(t)+D_{1} u\left(t-d_{2}(t)\right) \\
u(t) & =-K \xi(t) \\
\xi(t) & =\phi(t), t \in[-d, 0]
\end{aligned}
$$

Which $L \in R^{n \times p}$ is the observer gain and $K \in R^{m \times n}$ is the feedback control gain.

Let the error vector be such $e(t)=x(t)-\xi(t)$,

We combine (1) and (5) to produce the closed-loop system: 


$$
\begin{aligned}
& {\left[\begin{array}{ll}
E & 0 \\
0 & E
\end{array}\right]\left[\begin{array}{l}
\dot{x}(t) \\
\dot{e}(t)
\end{array}\right]=\left[\begin{array}{cc}
A_{1}+\Delta A_{1} & 0 \\
A_{1}-A_{D}+\Delta A_{1}-L \Delta C_{1} & A_{D}-L C_{1}
\end{array}\right]\left[\begin{array}{l}
x\left(t-d_{1}(t)\right) \\
e\left(t-d_{1}(t)\right)
\end{array}\right]} \\
& +\left[\begin{array}{cc}
A+\Delta A-B K-\Delta B K & (B+\Delta B) K \\
\left(A+\Delta A-B K-\Delta B K-A_{C}+B_{C} K\right)+L(\Delta D K-\Delta C) & \left(A_{C}+\Delta B K+B K-B_{C} K-L C-L N D K\right)
\end{array}\right]\left[\begin{array}{l}
x(t) \\
e(t)
\end{array}\right] \\
& +\left[\begin{array}{cc}
-\left(B_{1}+\Delta B_{1}\right) K & \left(B_{1}+\Delta B_{1}\right) K \\
-\left(B_{1}-B_{D}+\Delta B_{1}-L N D_{1}\right) K & \left(B_{1}-B_{D}+\Delta B_{1}-L N D_{1}\right) K
\end{array}\right]\left[\begin{array}{l}
x\left(t-d_{2}(t)\right) \\
e\left(t-d_{2}(t)\right)
\end{array}\right]
\end{aligned}
$$

Definition 1[5] For the uncertain system(1) and cost function(4), if there exist a control law $u(t)$ and a positive scalar $J^{*}$ such that for all admissible uncertainties, the closed-loop system(1) is asymptotically stable and the closed-loop value of the cost function(4)satisfies $J \leq J^{*}$, then $J^{*}$ is said to be a guaranteed cost and $u(t)$ is said to be a guaranteed cost control law of the uncertain system(1)

Lemma 1 Given matrices $Y ; H ; E$ of appropriate dimensions and with $Y$ symmetric, then $Y+H F E+E^{T} F^{T} H^{T}<0$ for all $E$ satisfying $F^{T} F \leq I$ if and only if there exists a scalar $\varepsilon<0$ such that $Y+\varepsilon H H^{T}+\varepsilon^{-1} E^{T} E<0$

\section{MAIN RESULTES}

Theorem 1 The closed-loop system(5) is robust stable and $u(t)=-K \xi(t)$ is a

guaranteed cost controller if there exist invertible matrices $P_{1}, P_{2}$ and symmetric positive-definite matrices $S_{1}, S_{2}, R_{1}, R_{2}$, he following matrix inequality holds

$$
\begin{aligned}
& E^{T} P_{i}=P_{i}^{T} E \geq 0, E^{T} R_{i}=R_{i}^{T} E \geq 0 \\
& {\left[\begin{array}{ll}
N_{1} & N_{2}^{T} \\
N_{2} & -\varepsilon I
\end{array}\right]<0}
\end{aligned}
$$

An upper bound on the cost $J^{*}$ is given by

$$
\begin{aligned}
& J^{*}=\left[\begin{array}{l}
\varphi(0) \\
e(0)
\end{array}\right]^{T}\left[\begin{array}{cc}
E^{T} P_{1} & 0 \\
0 & E^{T} P_{2}
\end{array}\right]\left[\begin{array}{l}
\varphi(0) \\
e(0)
\end{array}\right] \\
& +\int_{-\lambda}^{0}\left[\begin{array}{l}
\varphi(\sigma) \\
e(\sigma)
\end{array}\right]^{T}\left[\begin{array}{cc}
S_{1} & 0 \\
0 & S_{2}
\end{array}\right]\left[\begin{array}{l}
\varphi(\sigma) \\
e(\sigma)
\end{array}\right] d \sigma \\
& +\int_{-\eta}^{0}\left[\begin{array}{c}
\varphi(\sigma) \\
e(\sigma)
\end{array}\right]^{T}\left[\begin{array}{cc}
K^{T} R_{1} K & 0 \\
0 & K^{T} R_{2} K
\end{array}\right]\left[\begin{array}{l}
\varphi(\sigma) \\
e(\sigma)
\end{array}\right] d \sigma
\end{aligned}
$$

where,

$$
N_{1}=\left[\begin{array}{cccccc}
\Sigma_{1} & P_{1}^{T} A_{1} & -P_{1}^{T} B_{1} & \Sigma_{14} & 0 & P_{1}^{T} B_{1} \\
* & -(1-\alpha) S_{1} & 0 & \left(A-A_{D}\right)^{T} P_{2} & 0 & 0 \\
* & * & -(1-\beta) R_{1} & -\left(B-B_{D}\right)^{T} P_{2} & 0 & 0 \\
* & * & * & \Sigma_{4} & P_{2}^{T} A_{D}-P_{2}^{T} L C_{1} P_{2}^{T}\left(B_{1}-B_{D}\right) \\
* & * & * & * & -(1-\alpha) S_{2} & 0 \\
* & * & * & * & * & -(1-\beta) R_{2}
\end{array}\right]
$$

$N_{2}=\left[\begin{array}{cccccc}H_{1} & 0 & 0 & 0 & 0 & 0 \\ -H_{2} K & 0 & 0 & H_{2} K & 0 & 0 \\ 0 & H_{3} & 0 & 0 & 0 & 0 \\ 0 & 0 & -H_{4} & 0 & 0 & H_{4} \\ -H_{5} & 0 & 0 & 0 & 0 & 0 \\ H_{6} K & 0 & 0 & -H_{6} K & 0 & 0 \\ 0 & -H_{7} & 0 & 0 & 0 & 0 \\ 0 & 0 & H_{8} & 0 & 0 & -H_{8}\end{array}\right]$

$\Sigma_{11}=2(A-B K)^{T} P_{1}+S_{1}+K^{T} R_{1} K+Q+K^{T} R K+\varepsilon \sum_{i=1}^{4} P_{1}^{T} E_{i} E_{i}^{T} P_{1} ;$

$\sum_{14}=P_{1}^{T} B K+\left(A-A_{C}-B K+B_{C} K\right)^{T} P_{2}+\varepsilon \sum_{i=1}^{4} P_{1}^{T} E_{i} E_{i}^{T} P_{2} ;$

$\sum_{44}=2\left(A_{C}+B K-B_{C} K\right)^{T} P_{2}+S_{2}+K^{T} R_{2} K+\varepsilon \sum_{i=1}^{4} P_{2}^{T} E_{i} E_{i}^{T} P_{2}+\varepsilon \sum_{i=5}^{8} P_{2}^{T} L E_{i} E_{i}^{T} L^{T} P_{2}$

Proof. Consider the following Lyapunov function:

$V(x(t), e(t))=\left[\begin{array}{l}x(t) \\ e(t)\end{array}\right]^{T}\left[\begin{array}{cc}E^{T} P_{1} & 0 \\ 0 & E^{T} P_{2}\end{array}\right]\left[\begin{array}{l}x(t) \\ e(t)\end{array}\right]$

$+\int_{t-d_{1}(t)}^{t}\left[\begin{array}{l}x(t) \\ e(t)\end{array}\right]^{T}\left[\begin{array}{cc}S_{1} & 0 \\ 0 & S_{2}\end{array}\right]\left[\begin{array}{l}x(t) \\ e(t)\end{array}\right] d t$

$+\int_{t-d_{2}(t)}^{t}\left[\begin{array}{l}x(t) \\ e(t)\end{array}\right]^{T}\left[\begin{array}{cc}K^{T} R_{1} K & 0 \\ 0 & K^{T} R_{2} K\end{array}\right]\left[\begin{array}{l}x(t) \\ e(t)\end{array}\right] d t$

Then, the time-derivative of $V(x(t), e(t))$ gives

$\dot{V}+x^{T}(t) Q x(t)+u^{T}(t) R u(t) \leq \eta^{T}(t) \Omega \eta(t)$

where $\eta^{T}(t)=\left(x^{T}(t) x^{T}\left(t-d_{1}(t)\right) x^{T}\left(t-d_{2}(t)\right) K^{T} e^{T}(t) e^{T}\left(t-d_{1}(t)\right) e^{T}\left(t-d_{2}(t)\right) K^{T}\right)$ $\Phi_{11}$

$=(A-B K)^{T} P_{1}+P_{1}^{T}(A-B K)+S_{1}+K^{T} R_{1} K+Q+K^{T} R K ;$

$\Phi_{14}=P_{1}^{T} B K+\left(A-A_{C}-B K+B_{C} K\right)^{T} P_{2}-K^{T} R K ;$

$\Phi_{44}=2\left(A_{C}+B K-B_{C} K+L C\right)^{T} P_{2}+S_{2}+K^{T} R_{2} K+K^{T} R K ;$

$\Omega=\left[\begin{array}{cccccc}\Phi_{11} & P_{1}^{T} A & P_{1}^{T} B & \Phi_{14} & 0 & P_{1}^{T} B \\ * & -(1-\alpha) S & 0 & \left(A-A_{D}\right)^{T} P_{2} & 0 & 0 \\ * & * & -(1-\beta) & -\left(B-B_{D}\right)^{T} P_{2} & 0 & 0 \\ * & * & * & \Phi_{4} & P_{2}^{T} A_{D}-P_{2}^{T} L C_{1} P_{2}^{T}(B-B) \\ * & * & * & * & -(1-\alpha) S_{D} & 0 \\ * & * & * & * & * & -(1-\beta) R_{2}\end{array}\right]$

$+Q Q_{2} O_{3}+\left(O O_{2} Q_{3}\right)^{T}$

$O_{1}=\left[\begin{array}{cccccccc}P_{1}^{T} E_{1} & P_{1}^{T} E_{2} & P_{1}^{T} E_{3} & P_{1}^{T} E_{4} & 0 & 0 & 0 & 0 \\ 0 & 0 & 0 & 0 & 0 & 0 & 0 & 0 \\ 0 & 0 & 0 & 0 & 0 & 0 & 0 & 0 \\ P_{2}^{T} E_{1} & P_{2}^{T} E_{2} & P_{2}^{T} E_{3} & P_{2}^{T} E_{4} & P_{2}^{T} L E_{5} & P_{2}^{T} L E_{6} & P_{2}^{T} L E_{7} & P_{2}^{T} L E_{8} \\ 0 & 0 & 0 & 0 & 0 & 0 & 0 & 0 \\ 0 & 0 & 0 & 0 & 0 & 0 & 0 & 0\end{array}\right]$

$O_{2}=\operatorname{diag}\left(F_{1}(t), F_{2}(t), F_{3}(t), F_{4}(t), F_{5}(t), F_{6}(t), F_{7}(t), F_{8}(t)\right) ; O_{3}=N_{2}$ 
According to Lemma 1 and Schur Complement, it holds for any $F(t)$ satisfying (2),if and only if there exists a scalar $\varepsilon>0$, such that $\left[\begin{array}{cc}N_{1} & N_{2}^{T} \\ N_{2} & -\varepsilon I\end{array}\right]<0$.

Hence, $\quad \dot{V}<-\left[x^{T}(t) Q x(t)+u^{T}(t) R u(t)\right]<0$.

By (7),(8),(10) implies that system(5) is asymptotically stable.On the other hand, using (10), $\int_{0}^{T}\left[x^{T}(t) Q x(t)+u^{T}(t) R u(t)\right] d t<V(0)$, Let $T \rightarrow \infty$, we get (9).

Theorem 2 The closed-loop system (5) is asymptotically stable if there exist invertible matrices $X$ and symmetric positive-definite matrices $T, Y, M, N$, he following matrix inequality holds

$$
\begin{gathered}
E^{T} X^{-1}=X^{-T} E \geq 0, E^{T} T^{-1}=T^{-T} E \geq 0, E^{T} Y^{-1}=Y^{-T} E \geq 0 \\
{\left[\begin{array}{cccc}
T_{1} & T_{2} & T_{3} & T_{4} \\
* & -\mathcal{E} I & 0 & 0 \\
* & * & T_{5} & 0 \\
* & * & * & T_{6}
\end{array}\right]<0}
\end{gathered}
$$

An upper bound on the cost $J^{*}$ is given by (9). Where

$$
\begin{aligned}
& T_{1}=\left[\begin{array}{ccccccc}
\Pi_{11} & A_{1} M & -B_{1} T & \Pi_{14} & 0 & B_{1} Y \\
* & -(1-\alpha) M^{T} & 0 & M^{T} A_{1}^{T} & 0 & 0 \\
* & * & -(1-\beta) T^{T} & -T^{T} B_{1}^{T} & 0 & 0 \\
* & * & * & \Pi_{44} & 0 & B_{1} Y \\
* & * & * & * & -(1-\alpha) N^{T} & 0 \\
* & * & * & * & * & -(1-\beta) Y^{T}
\end{array}\right] \\
& T_{2}=\left[\begin{array}{cccccccc}
X^{T} H_{1}^{T}-W^{T} H_{2}^{T} & 0 & 0 & -X^{T} H_{5}^{T} & W^{T} H_{6}^{T} & 0 & 0 \\
0 & 0 & M^{T} H_{3}^{T} & 0 & 0 & 0 & -M^{T} H_{7}^{T} & 0 \\
0 & 0 & 0 & -T^{T} H_{4}^{T} & 0 & 0 & 0 & T^{T} H_{8}^{T} \\
0 & W^{T} H_{2}^{T} & 0 & 0 & 0 & -W^{T} H_{6}^{T} & 0 & 0 \\
0 & 0 & 0 & 0 & 0 & 0 & 0 & 0 \\
0 & 0 & 0 & Y^{T} H_{4}^{T} & 0 & 0 & 0 & -Y^{T} H_{8}^{T}
\end{array}\right]
\end{aligned}
$$

$T_{5}=\operatorname{diag}\left(-M,-T,-Q^{-1},-R^{-1}, \varepsilon_{1}^{-1} I,-\varepsilon_{2}^{-1} I, \varepsilon_{3}^{-1} I,-\varepsilon_{4}^{-1} I,-N,-Y\right.$,

$\left.-\varepsilon_{5}^{-1} I, \varepsilon_{6}^{-1} I, 2 C^{T} C,-\varepsilon^{-1} \sum_{i=5}^{8} C^{T} E_{i} E_{i}^{T} C,-\varepsilon_{7}^{-1} I, \varepsilon_{8}^{-1} I, \varepsilon_{9}^{-1} I\right) ;$

$T_{6}=\operatorname{diag}\left(\varepsilon_{1} I,-\varepsilon_{2} I, \varepsilon_{3} I, \varepsilon_{1} I,-\varepsilon_{4} I,-\varepsilon_{5} I, \varepsilon_{6} I,-\varepsilon_{7} I, \varepsilon_{8} I, \varepsilon_{9} I\right) ;$

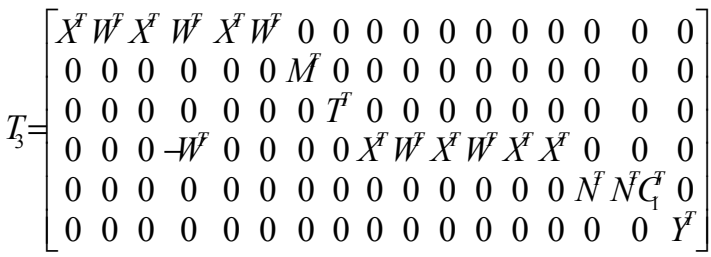

$T_{4}=\left[\begin{array}{ccccccccc}0 & 0 & 0 & 0 & 0 & 0 & 0 & 0 & 0 \\ 0 & 0 & 0 & 0 & 0 & 0 & 0 & 0 & 0 \\ 0 & 0 & 0 & 0 & 0 & 0 & 0 & 0 & 0 \\ A_{C} & B_{C} & A_{D} & B_{D} & A_{C} & B_{C} & A_{D} & B_{D} & X^{T} C^{T} \\ 0 & 0 & 0 & 0 & 0 & 0 & 0 & 0 & 0 \\ 0 & 0 & 0 & 0 & 0 & 0 & 0 & 0 & 0\end{array}\right]$

$\Pi_{11}=X^{T} A^{T}+A X-B W-W^{T} B^{T}+\varepsilon \sum_{i=1}^{4} E_{i} E_{i}^{T} ;$

$\Pi_{14}=B W+X^{T} A^{T}-W^{T} B^{T}+\varepsilon \sum_{i=1}^{4} E_{i} E_{i}^{T} ; \Pi_{44}=W^{T} B^{T}+B W+\varepsilon \sum_{i=1}^{4} E_{i} E_{i}^{T}$

Proof. Let $L=P_{2}^{-T} C^{T}, P_{1}=P_{2}$ Pre-multiplying and post-multiplying matrix inequality $(8)$ by $\operatorname{diag}\left(P^{-T}, S_{1}^{-T}, R_{1}^{-T}, P^{-T}, S_{2}^{-T}, R_{2}^{-T}, I\right)$ and $\operatorname{diag}\left(P^{-1}, S_{1}^{-1}, R_{1}^{-1}, P^{-1}, S_{2}^{-1}, R_{2}^{-1}, I\right)$,respectively.

Let $X=P^{-1}, M=S_{1}^{-1}, N=S_{2}^{-1}, T=R_{1}^{-1}, Y=R_{2}^{-1}, W=K P^{-1}$, hance, $K=W X^{-1}, L=X^{T} C^{T}$,then (12) is obtained by Schur Complement.

\section{CONCLUSION}

In this paper, the problem of observer-based guaranteed cost control for a class of singular time-delay systems with uncertainties has been studied. Sufficient conditions for the existence of guaranteed cost observer-based state feedback control law and corresponding guaranteed cost performance index are obtained. The results in this paper are much more desirable and less conservative than the existing results.

\section{REFERENCES}

[1] WU Zheng-guang; ZHOU Wu-neng.Delay-dependent Robust Stabilization for Uncertain Singular Systems with State Delay[J].Acta Automatica Sinica,Vol.33,No.7July,2007

[2] FANG Mei. Delay-dependent Robust $H_{\infty}$ Control for Uncertain Singular Systems with State Delay[J].Acta Automatica Sinica,Vol.35,No.1 January, 2009

[3] YANG Guang-hong. WANG Jian-liang and Yeng Chai Soch. Guaranteed Cost Control for Discrete-time Linear System under Controller Gain Perturbations . Linear Algebra and Application,2000,3(2): 161-180

[4] ZHANG Jin-hui;SHI Peng;QIU Ji-qing.Non-fragile Guaranteed Cost Control for Uncertain Stochastic Nonlinear Time-delay Systems[J].Science Diirect.Journal of the Frank Institute346(2009)676-690.

[5] YANG Fan;ZHANG Qing-ling.Guaranteed Cost Observer-based Control of Singular Time-delay Systems with Uncertainties[J].Control and Decision, 1001-09 20(2005)10-1177-04

[6] LI Hua; ZHOU Yu. Observer-based Non-fragile Guaranteed Cost Control of Singular Time-delay Systems[J].Control Engineering of China. 1671-7848 (20 10)06-0719-04 